\title{
Entre positionnements victimaires et attitudes identitaires : hétéro- et auto-désignations des Afrodescendants
}

Between victimhood and identity claims : Hetero- and auto-designations of Afrodescendants

\section{Sonia Branca-Rosoff}

\section{(2) OpenEdition Journals}

\section{Édition électronique}

URL : http://journals.openedition.org/aad/3624

DOI : 10.4000/aad.3624

ISSN : 1565-8961

\section{Éditeur}

Université de Tel-Aviv

\section{Référence électronique}

Sonia Branca-Rosoff, «Entre positionnements victimaires et attitudes identitaires : hétéro- et autodésignations des Afrodescendants », Argumentation et Analyse du Discours [En ligne], 23 | 2019, mis en ligne le 17 octobre 2019, consulté le 10 décembre 2020. URL : http://journals.openedition.org/aad/ 3624 ; DOI : https://doi.org/10.4000/aad.3624

Ce document a été généré automatiquement le 10 décembre 2020.

\section{(c) $(1) \subseteq$}

Argumentation \& analyse du discours est mis à disposition selon les termes de la licence Creative Commons Attribution - Pas d'Utilisation Commerciale - Pas de Modification 4.0 International. 


\section{Entre positionnements victimaires et attitudes identitaires : hétéro- et auto-désignations des Afrodescendants}

Between victimhood and identity claims : Hetero- and auto-designations of Afrodescendants

Sonia Branca-Rosoff

\section{Introduction}

1 En janvier 2017, des militants noirs dénoncent un scandale raciste à propos du projet de réouverture d'une salle de spectacle parisienne sous son ancien surnom de Bal nègre. Ils veulent obtenir un changement de nom en arguant que, quel que soit le contexte, "Nègre » est un mot insultant. La polémique connaît un pic après l'interview du propriétaire, Guillaume Cornut, dans la revue Africultures le 31 janvier 2017, la mise en ligne d'une pétition sur le site Change.org le $1^{\text {er }}$ février (\#NonAuBalDesColons! Pas de «bal nègre à Paris » en 2017¹), l'organisation d'une manifestation le 5 février devant l'établissement. Après une semaine de polémiques, le propriétaire retire le nom et adopte la dénomination de Bal Blomet. À partir de ce bref moment discursif, je me propose d'examiner quelques aspects de la construction des discours de ceux qui se dressent contre ce qu'ils nomment le « racisme structurel » qui, même en l'absence de lois discriminantes, entrave le développement des « Noirs ». Tantôt, ils en appellent à la compassion, tantôt ils dénoncent ceux qui, comme G. Cornut, paraissent aveugles au caractère blessant du signifiant "Nègre». J'aborderai aussi le contre-discours d'Antillais qui refusent de participer à la lutte pour un signifiant alternatif, et plus généralement toute posture victimaire. J'examinerai enfin les messages, que l'on trouve sur le site identitaire Riposte Laïque, de ceux qui se posent à leur tour en victimes d'un racisme « anti-Blancs ». 
2 J'adopterai un point de vue discursif qui part du fait que loin d'être strictement représentationnel, le langage sert à s'identifier, à construire des images sociales de soi et de l'autre. Cette position s'oppose à la perspective réaliste qui traite des catégories comme des faits autonomes préexistant à leur description. La position discursive, qui est par exemple celle du philosophe John Searle (1998), souligne la part langagière de la construction du monde social. L'analyse du discours, pour sa part, a conduit à s'intéresser particulièrement aux actes d'énonciation susceptibles de déplacer les représentations, soit que les sujets opèrent des choix parmi les dénominations disponibles (par exemple "Noir», "Black», à côté de "Nègre»)2, soit qu'ils infléchissent la valeur des dénominations en jouant sur le contexte. En partant des discours que les descendants des colonisés tiennent sur le racisme et qui permettent à des victimes de se constituer en force militante, je m'attacherai à la lutte menée contre l'usage du mot «Nègre » dans la dénomination Bal Nègre, les militants y voyant un signe qui conforte la perception péjorative des Noirs. Le propriétaire affirmait que ce mot a une valeur ambivalente, en particulier en ce qui concerne son orientation axiologique, ce qui est confirmé par un parcours dans les données de Frantext. Dans le cadre de ces conflits, les positions de victimes et d'agresseurs peuvent s'échanger: ainsi, de l'extérieur, on est frappé par la violence de l'attaque qui vise personnellement Cornut. Par ailleurs, dénoncés comme racistes, des «Blancs » revendiquent à leur tour une position de victimes.

\section{La politisation de la souffrance}

3 Je m'attacherai tout d'abord aux modalités énonciatives favorisant la formation d'un sujet collectif et à l'émergence de certaines figures de porte-parole. Les lecteurs sont invités à sympathiser avec les émotions et les raisons de ces leaders sortis de l'anonymat pour devenir rapidement des témoins des souffrances ressenties par les « Noirs » qui vivent dans les sociétés occidentales. De ce fait, ils se présentent comme des porteurs légitimes des revendications de ces communautés de victimes de l'histoire.

\subsection{Expression subjective de la souffrance engendrée par le racisme et établissement rationnel des faits dans l'essai de Reni Eddo-Lodge}

4 Depuis 2005, environ, moment qui a vu la création du mouvement des Indigènes ${ }^{3}$ de la République à la suite de la diffusion de l'appel « Nous sommes les Indigènes de la République !» et qui correspond à l'essor des réseaux sociaux, on voit se succéder des campagnes qui dénoncent les diverses formes de discrimination causées par l'idéologie postcoloniale ${ }^{4}$. Ceux qui s'expriment sur les réseaux sociaux et dans les médias comparent la situation actuelle des minorités ethniques avec celle des esclaves ou celle des indigènes des colonies, et demandent à ce que les États européens réparent les torts causés aux victimes du passé colonial et du présent postcolonial.

5 Ce mouvement émerge dans une période marquée par la montée des inégalités nées de la mondialisation néolibérale, par l'obsolescence rapide des techniques industrielles qui entraîne l'accroissement du chômage. L'exclusion sociale qui en découle ruine le sentiment d'appartenir à une république commune. L'impuissance des États, l'inefficacité des organisations syndicales et politiques traditionnelles, entrâ̂nent la 
méfiance envers les responsables politiques qui n'apparaissent plus que comme des notables illégitimes. Les nouvelles technologies de l'information et de la communication permettent aux dénonciations de se déployer rapidement hors des structures traditionnelles des mouvements politiques, aux militants de se connecter avec les mouvements des minorités aux États-Unis, d'établir des liens étroits avec des militants en Afrique ou dans les départements et régions d'outre-mer. Par ailleurs, les leaders se retrouvent dans le CRAN qui comprend une soixantaine d'associations, notamment des associations représentant des Africains et des Antillais vivant en France 5 .

6 Dans un certain nombre d'essais récents, les Noirs qui, s'estimant victimes du racisme, prennent la parole, passent du témoignage sur leur expérience vécue au problème général du racisme. L'articulation du cas singulier et du significatif est typique de l'essai de Reni Eddo-Lodge (2018), Le Racisme est un problème de blancs ${ }^{6}$. Au-delà de son aspect documentaire, le texte permet d'entrer dans l'intimité de l'auteure et de comprendre un peu mieux la virulence de conflits autour de faits qui, de l'extérieur, pourraient paraître mineurs. Le livre décrit d'abord sa colère en face du manque d'empathie des Blancs. Enfermés dans le déni de la réalité qu'elle vit, ils en viennent à la considérer comme une malade ou comme une folle.

Je n'en peux plus du détachement vertigineux qu'ils [les Blancs] affichent quand une personne de couleur raconte son vécu [...] Sans compter que discuter avec les Blancs arrogants est en soi un exercice franchement périlleux pour moi. A mesure que le ton monte et se fait plus agressif il me faut redoubler de maîtrise. Car si j'exprime la moindre frustration, irritation ou exaspération face à leur refus de comprendre, ils ressortiront leurs discours racistes prémâchés sur les Noirs en colère qui les menacent eux et leur sécurité. C'est moi bien sûr qui serais tyrannique, agressive (2018: 9-11).

7 La suite raconte son expérience intime et quotidienne des regards posés sur elle qui la ramènent toujours à sa couleur de peau, souffrance qui est une clé pour expliquer la persistance des inégalités: "J'ai écrit ce livre pour dire ce que ça fait de se voir arracher sa voix et sa confiance face à un inébranlable statu quo » (17).

Dans un témoignage, il y a un premier aspect qui est d'attester que des faits se sont produits. Mais pour l'essentiel, un témoignage est chargé d'affects. Il rend compte des effets subjectifs des expériences vécues sur la personne. On ne peut donc lui opposer d'arguments « objectifs ». Il n'y a rien à objecter quand une jeune femme évoque les sentiments destructeurs que provoquent les réflexions ou les gestes de ses interlocuteurs blancs. Que répondre quand elle explique que l'Angleterre postcoloniale lui est insupportable et que, dès qu'elle se plaint, elle est considérée comme "une femme noire en colère obsédée par les questions raciales» $(2018: 120)$ ? La seule réponse est la sympathie qui, selon Les Lumières ${ }^{7}$, est une faculté anthropologique permettant de s'identifier au malheur des victimes. Manifester publiquement sa souffrance, c'est l'adresser à des lecteurs capables de devenir des partenaires pour qu'ils la comprennent, qu'ils perçoivent le caractère dévastateur de ces expériences traumatiques et sortent de leur indifférence.

Mais peut-on considérer qu'il s'agit de racisme dès lors que les offenses sont «imperceptibles»:

Le terme [racisme] structurel est souvent le seul moyen de désigner ce qui est imperceptible: les froncements de sourcils silencieux, les préjugés implicites, les jugements à l'emporte-pièce, sans fondement réel, sur la compétence d'une personne $\left(87^{8}\right)$. 

objecter que sa douleur ne vient pas d'un problème de racisme, mais d'une hypersensibilité personnelle qui ne saurait donner lieu à une réponse politique. Il pourrait même accuser l'auteure de tirer une rente d'un handicap supposé qui ne l'a pas empêchée de faire une carrière médiatique. La demande d'empathie se révèlerait alors contre-productive.

Il est donc essentiel de montrer que ces émotions correspondent à des faits historiques bien établis, que l'expérience rapportée est exemplaire. C'est ce que font les chapitres 2 et 3 du livre qui inscrivent le cas individuel dans un contexte collectif, qui le "désingularisent ${ }^{9}$ en décrivant l'histoire récente des Noirs en Grande-Bretagne. Malgré leur participation aux deux guerres mondiales, ils subissent de nombreuses discriminations, des attaques racistes, des contrôles policiers donnant lieu à de multiples bavures. Le récit subjectif de Reni correspond aux savoirs sur la discrimination empruntés aux historiens et aux sociologues: parallèlement, l'énonciation subjective laisse place à un effacement de l'énonciateur.

11 Cette articulation entre passion et raison permet de politiser la souffrance, de la transformer en cause. Elle est souvent à l'arrière-plan de témoignages où des victimes rejoignent un groupe militant pour donner une dimension collective à la lutte contre les inégalités qu'elles attribuent au racisme persistant.

\subsection{Les réseaux sociaux : un amplificateur de colère et une invitation à l'action}

Ce récit d'expérience d'une Européenne noire aurait pu rester cantonné à une sphère communautaire restreinte, mais, comme le raconte Reni Eddo-Lodge dans sa préface, le texte-source de son livre, posté sur les réseaux sociaux, a été massivement partagé. Plus généralement, c'est bien à l'articulation des textes des intellectuels et de leur circulation sur internet que se développent des formes inédites de mobilisation (commentaires et partage sur les réseaux, pétitions, manifestations non organisées par des appareils politiques traditionnels, etc.) autour d'une contestation radicale du politiquement correct issu de l'après-guerre, en particulier de l'interdit langagier formulé autour du thème de la race.

Régis Debray a souligné depuis longtemps l'effet déterminant des médias qui changent l'espace et le temps où se forgent les opinions. Sur le net, les messages se propagent de façon illimitée et ils se diffusent extrêmement rapidement. Leur répétition fait que (au moins sur les réseaux) des voix minoritaires dans un pays apparaissent comme dominatrices. La diffusion permet donc la convergence des positions. Gabriel Tarde (1843-1904) déjà propose de donner un statut sociologique à la fabrique des identités sociales en définissant le groupe social comme " une collection d'êtres en tant qu'ils sont en train de s'imiter entre eux ou en tant que, sans s'imiter actuellement, ils se ressemblent et que leurs traits communs sont des copies anciennes d'un même modèle » (1979: 73). La perspective de Tarde oriente la recherche dans une direction sensible à la dimension active et interactive du discours. Si l'on applique ses réflexions aux activités qui se développent sur les réseaux sociaux, on voit que des énoncés qui paraissaient isolés et provocateurs deviennent "réguliers" dans certains de ces réseaux (au double sens de leur fréquence et de leur caractère normatif). C'est pourquoi

Argumentation et Analyse du Discours, 23 | 2019 
nous tenons compte dans les passages suivants des commentaires qui circulent sur les réseaux, tout autant que des « textes-sources".

Des porte-parole ${ }^{10}$ émergent dans ce contexte. En France, les plus connus sont Patrick Lozès, docteur en pharmacie, fondateur et premier président du CRAN ; Pap N'Diaye, professeur à l'IEP de Paris, qui a fondé avec Patrick Lozès le Cercle d'action pour la promotion de la diversité en France (CAPDIV) ; Louis-Georges Tin, ancien élève de l'ENS et Maître de conférences à l'université d'Orléans ; plus récemment, Rokhalla Diallo, journaliste et essayiste. Tous ont en commun de coupler bataille socio-politique à long terme et buts limités (donc) atteignables dans une action immédiate. A chaque dérapage qu'ils jugent raciste, ces intellectuels militants développent un activisme numérique mobilisateur. La mobilisation autour des offenses racistes ne rassemble pas toujours un nombre important de personnes, mais lorsqu'elles sont reprises, les protestations se propagent rapidement et donnent une visibilité énorme aux thèses du mouvement. J'aurais pu m'intéresser à d'autres mises en cause comme le refus de la lexie « nègre littéraire », l'appel à la suppression de l'enseigne Au Nègre joyeux dans la rue Mouffetard, le remplacement du nom des gâteaux dits " têtes de nègre ", etc., mais je m'en tiendrai à la controverse qui a eu lieu en 2017 autour de l'appellation Bal Nègre que Guillaume Cornut, son nouveau propriétaire, voulait donner à sa salle de spectacle.

Il s'agit moins de savoir si les protestataires ont raison ou non, que de se demander ce que "font» les notions qu'ils font circuler sur les réseaux à la fois en termes d'imposition de catégories socio-historiques nouvelles (racialisé = perçu comme une race ; racisés = discriminé en fonction de sa race supposée, racisation ${ }^{11}$, décolonial), en termes d'émergence de minorités et d'affirmation de nouveaux leaders d'opinion ${ }^{12}$.

\section{L'affaire du Bal de la rue Blomet}

On trouve sur le site du Bal Blomet (http://www.balblomet.fr/histoire-bal-blomet/) une présentation de l'histoire de l'établissement qui peut être complétée par le livre de Brigitte Léardée et Jean-Pierre Meunier (1989), La Biguine de l'Oncle Ben's: Ernest Léardée raconte, ainsi que par les souvenirs des nombreux artistes et intellectuels qui s'y retrouvaient, notamment ceux de Brassaï dans son Paris secret des années trente (1978).

\subsection{Une brève histoire du Bal Blomet}

17 L'affaire du Bal situé au 33 de la rue Blomet dans le 15e arrondissement, commence vraiment en 1931 lorsque le poète surréaliste Robert Desnos, qui habite quelques mètres plus loin dans un atelier d'artiste, le renomme Bal Nègre et en assure la promotion dans un article de la revue Comoedia ${ }^{13}$ :

Dans l'un des plus romantiques quartiers de Paris, où chaque porte cochère dissimule un jardin et des tonnelles, un bal oriental s'est installé. Un véritable bal nègre où tout est nègre, les musiciens comme les danseurs : et où l'on peut passer, le samedi et le dimanche une soirée très loin de l'atmosphère parisienne parmi les pétulantes Martiniquaises et les rêveuses Guadeloupéennes. C'est au 33 de la rue Blomet, dans une grande salle attenante au bureau de tabac Jouve, salle où, depuis bientôt un demi-siècle, les noces succèdent aux réunions électorales. (https:// fr.wikipedia.org/wiki/Bal_N \%C3 \%A8gre =)

18 Le bal, comme l'écrit Desnos, a d'abord été un lieu de réunion politique. En 1924, Jean Rézard des Wouves, candidat antillais à la députation, y avait installé son quartier 
général, dans un commerce de vins. Les réunions électorales se sont vite transformées en soirées dansantes quand Jean Rézard, qui n'avait guère de public en tant qu'orateur politique, a eu l'idée d'y jouer la biguine de ses origines. Le bal devint un point de rencontre pour les Antillais, très vite rejoints par le Paris branché attiré par l'exotisme, l'érotisme et les rythmes entraînants du lieu. Ernest Léardée, violoniste et directeur de l'établissement pendant quelques années insiste sur l'espace de rencontres que constituait le Bal Blomet : « il était devenu à la mode d'aller danser chez les Noirs et pas un étranger ne quittait Paris sans être venu passer au moins une soirée dans ce lieu inhabituel où se mélangeaient toutes les races et toutes les nationalités » (Léardée et Meunier 1989 : 17).

Durant la Seconde Guerre mondiale, les Allemands interdisent le bal qui, après la guerre, ne retrouvera pas l'aura d'antan. L'établissement était menacé de démolition quand l'ex-tradeur et pianiste Guillaume Cornut rachète la salle avec l'intention d'y programmer du jazz et de la musique classique. Cornut avait pensé reprendre à Desnos l'appellation Bal Nègre pour, selon lui, rester fidèle à une époque qui avait découvert et exalté les cultures africaines. Le problème, c'est que ce discours valorisant n'a pas effacé la mémoire des discours coloniaux, ni les rapports sociaux d'oppression qui la sous-tendent. Rokkaya Diallo lance une pétition soutenue par le Cran pour faire retirer ce nom. Dialogue impossible : le propriétaire veut voir seulement le discours culturel, les militants du Cran voient seulement le discours colonial.

\subsection{La pétition}

\#NonAuBalDesColons! Pas de « bal nègre à Paris » en 2017

Nous sommes en 2017. M. Guillaume Cornut a décidé d'ouvrir prochainement un cabaret au 33, rue Blomet dans le 15ème arrondissement de Paris et de le baptiser tout simplement «Le Bal Nègre». Le concepteur du lieu puise de manière totalement assumée dans le registre de la nostalgie coloniale. Son site internet décrit l'époque des années 1920 ainsi : «La génération des Années folles est alors avide de distractions sur fond de musique et rêve d'un monde nouveau en réaction aux souffrances de la Grande Guerre. On se passionne frénétiquement pour les cultures inédites et les nouvelles esthétiques comme le Surréalisme, Dada, le Jazz ou l'art nègre qui culmine avec l'Exposition coloniale de 1931 ». Les zoos humains, où étaient exposés les colonisés, sont donc pour lui le point «culminant » d'une joyeuse époque. Peut-on raisonnablement célébrer ainsi une époque qui fait écho aux douleurs des descendant.e.s de colonisé.e.s? Le mot nègre est offensant pourtant le créateur du lieu en fait une utilisation emphatique et délibérée dont il semble même se vanter. Dans une interview donnée à France Info, il évacue l'aspect polémique du nom offensant du lieu d'une manière désinvolte : Restait le nom de la salle, qui risquait de choquer. Guillaume Cornut a donc consulté les Antillais de Paris, comme l'écrivain guadeloupéen Claude Ribbe. Verdict: "C'est un nom qui appartient à la salle, à son histoire. Il fait honneur à la communauté antillaise, qui est très fière de ce lieu ». Le Bal restera donc Nègre et la fête sous les auspices du piano du candidat député Rézard des Wouves. Qui sont ces «Antillais de Paris»? Mystère. Qui peut raisonnablement croire qu'une instance représentative des Antillais de Paris s'est réunie pour adouber ce lieu? Nous avons pris contact avec Claude Ribbe qui nous a fermement indiqué qu'il n'avait jamais tenu ces propos et tient à faire savoir qu'il se désolidarise totalement de cette initiative, dont il ne cautionne ni le nom ni le projet.

Nous sommes en 2017 et regrettons de devoir recourir à une pétition, aux réseaux sociaux pour rappeler une évidence : « Nègre » est un terme raciste. Interrogé sur le sens du mot M. Guillaume Cornut répond avec désinvolture «Je ne me suis pas 
documenté sur ce mot [...] J'en suis resté à ce qu'étaient les années 20 ». Il propose ainsi sans aucun recul historique une rhétorique coloniale datant de près d'un siècle ! Ce terme est pour nous une insulte raciale. Pourquoi en 2017, en France au pays des droits humains, une enseigne aussi insultante peut-elle avoir pignon sur rue et ce sans que la Ville de Paris ne trouve absolument rien à y redire ? Bien au contraire. Ce projet est soutenu par la Ville de Paris, alors que les artistes racisé.e.s peinent à disposer d'espaces pour se produire! Cornut n'a même pas pris la peine d'impliquer des personnes noires dans la conception de son projet. Ce faisant il ne rend hommage pas à la culture afro-américaine comme il le prétend mais injurie des millions de Noires et Noirs de France ${ }^{14}$. Nègre) et de la mémoire des discours sur l'art afro-américain qu'il convoque. Il faut aussi éviter de confondre le sémantisme franchement insultant de Nigger et celui de « Nègre ». Ces nominations ne sont pas porteuses de la même histoire :

Attention, on parle d'une expression - «Le Bal Nègre " - pas d'un mot isolé. Si j'avais baptisé le lieu "Le Bal Blomet», j'aurais eu des critiques comme quoi je récupère le lieu et que du coup ça disparaît de la mémoire. Et puis, vous savez, je 
pense à Aimé Césaire, à Senghor et son poème "Femme Noire" [Il récite les premiers vers du poème]. La poésie a écrit des choses splendides sur cette couleur! Même si ce mot est une insulte pour les Américains, il faut remettre ce nom dans son histoire, son origine, plutôt qu'essayer de le faire disparaître. C'est le racisme qu'il faut faire disparaître, pas le mot « nègre »! (Interview donnée à Africultures le 31/1/2017, voir Sadaï 2017) Haïtiens, présente un héros noir, modèle de grandeur héroïque ${ }^{19}$. A l'affrontement entre esclaves et blancs, correspond bien une dépréciation du terme « Nègre ». Un des protagonistes, un métis, heureux d'humilier un «Blanc », l'interrompt ainsi : « Nègres et mulâtres! Qu'est-ce que cela veut dire? Viens-tu ici nous insulter avec ces noms odieux, inventés par le mépris des blancs ? Il n'y a ici que des hommes de couleur et des noirs » (1826: 152). Plus tard, dans William Shakespeare (1864), Hugo présente la même opposition : « Jaloux, le héros est vite monstre! Le noir devient nègre. Comme la nuit a vite fait signe à la mort !» $(1864: 364)$

31 Victor Hugo confirme donc l'affinité de « Nègre " pour le pôle négatif, quand le terme est opposé à « Noir ». Mais le même Hugo témoigne de la neutralité de « Nègre » dans d'autres contextes. Dans une lettre au rédacteur en chef du Progrès, M. Heurtelou, un 
Haïtien, Hugo le désigne comme "Nègre ", alors qu'il ne cherche certainement pas à offenser son destinataire : «Il n'y a sur la terre ni blancs ni noirs il y a des esprits ; vous en êtes un. [...] Il est beau que parmi les flambeaux du progrès, éclairant la route des hommes, on en voit un tenu par la main d'un nègre» (lettre du 31 mars 1860, O. C. vol. $10: 728)$.

" Nègre " est certes employé comme insulte avec des contextes comme "espèce de ", "sale », mais il suffirait de mettre "Noir » dans de tels contextes pour déclencher la connotation offensante. Par ailleurs, même une fois que l'esclavage est aboli, la colonisation qui se développe à partir du Second Empire est justifiée en quelque sorte par les stéréotypes racistes associés aux Africains. Quels que soient les termes employés, l'Africain est alors associé à des jugements de valeur très dépréciatifs. Il faut attendre le début du 20e siècle pour voir un début de renversement, d'abord dans le milieu artistique : Paul Gauguin est, dès 1886, le premier à s'inspirer de l'art des peuples dits primitifs ${ }^{20}$. Il est suivi dans les premières années du $20^{\mathrm{e}}$ siècle par Georges Braque, Henri Matisse, André Derain et bien sûr Picasso qui cherchent dans les masques africains les clés d'une simplification des formes. La musique de l'Afrique et des Antilles triomphe à partir de 1925 et surtout avec La Revue nègre et Joséphine Baker dans le rôle principal qui impose le double exotisme (et érotisme) de l'Amérique et des Noirs. En 1927, un ancien tirailleur sénégalais fonde le journal antiimpérialiste La Voix des nègres. Dix ans plus tard, Aimé Césaire forge le terme "négritude» dans un article où il revendique l'identité noire et sa culture, contre la culture française trop cartésienne.

C'est cette ambivalence que la pétition veut liquider ce qui ne va pas sans conflits, comme le montrent les discussions publiées par Africulture ${ }^{21}$ et par les États Généraux de l'Outre-Mer (l'orthographe et la syntaxe n'ont pas été corrigées). Le texte du Cran qui affirme "Nègre est un terme raciste" a une valeur dialogique évidente puisqu'il s'oppose à la thèse antagonique de Cornut. De fait, en contexte, une affirmation peut n'avoir pas seulement une valeur descriptive, mais avoir pour but polémique de réduire au silence l'adversaire ${ }^{22}$. A ce dialogue sous-jacent, s'ajoute la dynamique des échanges sur le net. Une opinion n'a en en effet d'importance que si elle est adossée à une opinion favorable. Or, la polémique du Bal Blomet réactualise des débats anciens. En 2009, les internautes qui s'expriment sur le site des Etats Généraux de l'Outre-Mer se divisent entre ceux qui veulent bannir le mot et ceux qui se réclament de Césaire et de Senghor :

Je dis bien: Classer le mot «nègre » dans la catégorie des expressions à très forte connotation raciste, de manière claire et définitive. Et oui le racisme est punissable en France. C'est bien ! Il faut faire attention à ce qu'on dit : on insulte pas les gens et on n'accepte pas les insultes c'est ça l'attitude de l'homme digne, c'est ce que mon papa m'a dit ha ha! et à bien regarder c'est la Négritude qui est en fin de compte une interminable pleurnicherie, notre Histoire est bien ce qu'elle est et la prendre comme elle est! il faut faire face à la vie et surtout ne pas biaiser. Amicalement ! Gabriel Bavarday (États Généraux de l'Outre-Mer. Bannir le mot nègre du vocabulaire. 08/07/2009).

La controverse met surtout aux prises Gabriel Bavarday et Pascaline qui revendique au contraire "Nègre » en rappelant le sens positif du mot en créole et qui convoque Césaire et Senghor dont l'exemple montre qu'on peut inverser l'orientation axiologique d'un mot et le transformer en signe identitaire. Le renversement de l'insulte en autodésignation fièrement revendiquée est d'ailleurs assez fréquent. On peut citer les exemples anciens des «sans-culottes » ou des « impressionnistes » ou l'exemple actuel des jeunes gens d'origine populaire qui adoptent le mot « racaille» comme étendard. 
Dans tous ces cas, la reprise du désignant péjoratif qui circule dans l'opinion et son inversion axiologique s'accompagnent d'une invitation à se solidariser autour de ce nom appelé à devenir un signe identitaire. Aurelio Principato (2016) parle à ce propos de « réversibilité cohésive ».

Si l'on interdit le mot nègre, c'est que l'on copie bêtement la culture anglo-

saxonne ${ }^{23}$ et l'on oublie nos grands intellectuels afro comme Aimé Césaire. « Oui je suis nègre et alors?»

Je pense que l'on devrait apprendre à nos enfants à ne pas avoir honte de ce mot. Et d'en comprendre tout le sens pour pouvoir relever la tête face aux insultes racistes. Un nègre c'est celui qui a su survivre à la traversée de l'Atlantique dans des conditions atroces, puis survivre à des conditions de travail et des humiliations inimaginables pour enfin devenir ce qu'il est aujourd'hui un afro libre et fière.

Alors « Oui je suis nègre et alors? » Mon conseil apprend à vivre avec ton passé et à assumer... afin de clouer le bec des « racistes » (Pascaline, Guadeloupe, 04/06/2009).

En réduisant le mot "Nègre » à une insulte raciste, ceux qui veulent le censurer ne tiennent compte ni de la dynamique de l'émancipation, ni de l'opinion de ceux qui ne veulent pas réduire leur identité à un combat pour une reconnaissance victimaire.

Ah parce que c'est le courant de pensé qui te dérange!

Certes Césaire et Diop ne sont pas de ma génération, mais je trouve que leur travaux étaient nécéssaire pour faire de moi ce que je suis aujourd'hui. Le monde se construit sous forme de palier. Il y a différents paliers pour arriver à un certain stade. Le palier négritude était nécéssaire dans la francophonie pour justement être un autre modèle que celui proposé par les anglo saxons. Il a fallu plus de temps moins de censure au modèle de la négritude mais au moins le résultat est là. On est ce que l'on est, et l'on a pas honte de l'affirmer et l'on a pas honte de ce mot. « nègre » avec mes cheveux grainés. « nègre » avec mon goût prononcé pour la musique. "nègre » avec cette piqure du passé pour me rappeler à chaque instant que je ne devrai pas agir de façon raciste envers un autre. [..] 1 y a des choses bien plus importante au niveau économique et sociale à régler aux Antilles avant de vouloir détruire le travail identitaire de certains anciens.

Marquer la rupture avec la poésie sur la négritude, c'est montrer qu'un mot n'a pas d'importance pour nous, ainsi ce qui compte c'est notre avenir. Developpement durable, croissance durable, égalité, fraternité et surtout liberté ... ! Les anciens se sont arrêtés à la négritude nous on va essayer d'aller plus loin. C'est pas un mot qui va changer notre environnement économique et social (Pascaline. Guadeloupe 08/07/2009).

36 Les termes du débat n'ont pas changé en 2017. En en prenant connaissance, on s'aperçoit que la cible visée par le Cran est double, non seulement les Blancs et leur bonne conscience, mais aussi ceux des Noirs à qui le Cran reproche de s'enfermer dans une résignation sans issue, là où les nouveaux militants préfèrent inciter à la révolte :

[Nègre] c'est un très beau mot, et les Antillais l'emploient à merveille, tandis que d'autres en ont honte. Est-ce qu'ils en ont honte parce qu'on y entend négritude? Rouvrir le lieu avec son nom d'origine était rendre hommage aux musiciens antillais qui l'ont fait vivre, ne pas les oublier. Le Bal Nègre n'avait justement rien à voir avec l'exposition coloniale, ouvert par un Antillais, tenu par des Noirs, ouvert à tous, sans distinction, il était l'antithèse des zoos humains que dénonçaient les surréalistes. Je regrette qu'on soit parvenu à force de pressions à faire abandonner le nom. Finalement, le nègre a été interdit de bal. Est-ce une victoire? J'y vois un blanchiment d'enseigne 迤部 (Jean, le 8 février 2017).

37 Ainsi, la polémique concerne aussi les luttes politiques pour le contrôle de la communauté noire. Quant à Cornut, il a sagement choisi la désescalade en acceptant de renoncer au premier nom qu'il avait choisi. Le Bal Blomet a ouvert dans le calme. Bref, 
le mot "Nègre" dans un contexte non raciste ne devrait pas être offensant, mais les affrontements autour du Bal Blomet, et ceux qui ont précédé ou suivi, ont focalisé l'attention sur ce mot, fait entendre la voix de ceux qui se sentent insultés, et ce refus qui s'incorpore à la mémoire collective contribue à renforcer la gêne qu'il suscite.

\subsection{Noirs et Blancs : une opposition binaire entre des collectifs de victimes et de coupables}

Le mot « Nègre » est récusé comme raciste, mais son substitut, "Noir », se réclame d'une vision raciste inversée qui permet aussi de montrer les enjeux du travail lexical. Reni Eddo-Lodge dans son essai explique bien les enjeux du travail de redéfinition des collectifs qu'implique la construction de la catégorie des "Noirs ». Il s'agit d'imposer des catégories politiques de victimes: celle des "Non-Blancs", en incluant les Asiatiques ou les Arabes, etc., permet de faire reconnaître les torts que les esclavagistes et les colonialistes ont fait subir à des populations infériorisées... Celle des « Noirs » permet de refuser le continuum physique entre « Noirs » et «Blancs» et de renvoyer les métis dans le groupe des « Noirs ». Le paradoxe est que cette position reconduit la conception raciste des États-Unis pour qui une goutte de sang noir fait de vous un « Noir» (One drop Rule). Reni met d'ailleurs en garde contre les difficultés identitaires suscitées par les mariages mixtes. Une discussion posée comme emblématique l'amène à suggérer que l'identification d'une jeune fille métisse comme noire se heurte selon cette dernière à un déni de la part de la partie blanche de la famille qui n'a pas su prendre en compte l'impact de la race sur la vie des enfants. Plus généralement, « [la] croissance du métissage dans le pays complique les relations raciales plus qu'elle ne les simplifie [..] avec la hausse du nombre de familles mixtes et d'enfants métis les vifs débats relatifs à la race et au privilège blanc se retrouvent désormais au cœur des foyers (Eddo-Lodge, 129-130).

L'opposition binaire des victimes racisées et des "non-racisés " (euphémisme pour Blancs) met l'accent sur les ressemblances et rend aveugle aux différences (en particulier celles qui séparent les capitalistes et les prolétaires ou bien les hommes et les femmes). La force du langage consiste à simplifier, à réduire la multiplicité des identités, à fabriquer des ensembles séparés à forte connotation axiologique, dans le cas du Bal Blomet, en démarquant un groupe de Noirs "racisés ", ce qui explique leur statut économique et social bas et renvoie toute la responsabilité de l'inégalité aux Blancs ou "Souchiens »). La victimisation ouvre un espace de contestation des règles établies (par exemple, les concours méritocratiques) en soutenant qu'ils sont le masque des privilèges que s'octroient les Blancs et que la communauté discriminée a le droit d'obtenir le contournement de ces règles.

Si l'on écarte toute causalité complexe pour fonctionner par couples antithétiques, on débouche presque inévitablement sur la constitution symétrique d'un groupe de «Blancs». Assignés à la place de "coupables», les «Blancs» se voient imputer collectivement l'entière responsabilité de la situation des « Noirs ». La même opération de simplification passe sous silence les complexités de l'histoire et la multiplicité des situations dans la société actuelle. Ces demandes provoquent en retour un contrediscours extrêmement violent. Protégés par l'anonymat d'internet, des participants du site Riposte Laïque se décrivent à leur tour comme des victimes menacées par des envahisseurs africains. A l'identité blessée, ils opposent leur identité menacée, en 
demandant qu'on protège les détails symboliques (gâteaux Bamboula ou têtes de nègres "bien-de-chez-nous ») qui exaspèrent justement les antiracistes :

Quand j'etais gamin on pouvais acheter des tetes de Negres dans les boulangeries et des Biscuits Bamboula (marque St Michel) dans les grandes surfaces!!! J'aimais bien moi tout ça! Si ça continu, on aura meme plus le droit de les regarder, sans etre accuse de racisme ?!!!!! Il y en a ras le cul! On peut plus rien faire ou rien dire dans ce pays, ces gens la font la loi! (antigauche, https://ripostelaique.com/ louis-georges-tin-veut-interdire-a-un-ecrivain-noir-de-prendre-un-negreblanc.html)

41 Ils refusent d'accepter la France multiethnique et multiculturelle telle qu'elle est. La métaphore de la réserve exprime leur rêve d'une société fermée qui pourrait exclure les nouveaux-venus... Le mot "réserve » évoque curieusement l'Afrique et donc le retournement symbolique de cette lutte victimaire. Les «Blancs » de la dernière tribu (gauloise ?) rêvent de tracer une frontière contre les barbares, musulmans ou « Noirs ", qui menacent leur identité éternelle :

La moindre des choses serait d'exiger de nos corrompus des réserves de Français sur notre territoire, des réserves ethniques $100 \%$ Françaises, gérées par des Français, loin de l'ethnocide actuel. 30 \% d'Africains en France c'est trois fois plus de colons subi dans n'importe quelle colonisation au monde. Je suis certain que beaucoup de Français seraient ravis de vivre leur vie dans de petites réserves loin des us et coutumes des occupants et interdite à ceux qui n'y sont pas invités. Avoir le droit à une petite parcelle de son propre pays colonisé et ethnocidé, c'est la moindre des choses. Cela figure dans la déclaration universelle des droits de l'homme, liberté de conscience, droit à l'autodétermination etc...Sans Tin, Obonno, Bouteldja, Diallo etc... LE BONHEUR! (Crixus, 22/11/2017, https:// ripostelaique.com/louis-georges-tin-veut-interdire-a-un-ecrivain-noir-de-prendreun-negre-blanc.html)

42 Le problème du renversement opéré par les contributeurs de Riposte Laïque pour obtenir le statut de victime est qu'ils se heurtent aux inégalités constatées par les Noirs alors que la République qu'ils sont supposés défendre se réclame de l'égalité. Il ne reste plus qu'à frapper les esprits en laissant de côté les arguments rationnels et à s'adresser aux émotions du lecteur.

\subsection{Le rôle des émotions}

43 Ainsi, dans le discours victimaire, le recours à l'émotion a un caractère de nécessité. L'émotion peut être dite verbalement comme dans le texte du Cran " Le mot nègre est offensant». Cornut "injurie des millions de Noires et Noirs de France», ou dans le commentaire de Ceecee sur « ce que les Afro-descendants peuvent ressentir »:

Malheureusement, la France se fiche de ce que les afro-descendants peuvent ressentir. Nous sommes des paranoïaques qui voyons le mal partout [...] Je suis toujours circonspecte de voir comment une personne non concerné par l'offense (utilisation du mot «nègre » à visée commerciale) peut s'approprier ce mot et n'y voir aucune malice [...] il y a un deux poids deux mesures qui est assez hallucinant mais tellement banale dans ce pays que l'ont fini par juste soupirer et espérer que les afro-descendants se révoltent physiquement un jour (Ceecee, 31/1//2017, Africultures)

Elle est souvent spectacularisée, comme dans certains commentaires d'Afro-fem où l'on peut noter le recours à des énoncés courts sans prédicat pour décrire la situation, l'emploi d'énoncés injonctifs (« méfions-nous), de vocatifs (« M. Cornut sachez »), d’une 
ponctuation exclamative. Des mots grossiers qui agressent violemment l'adversaire peuvent apparaître. Les émotions sont alors « montrées » et non plus racontées :

« Racialisé » du matin au soir. Aujourd'hui en 2017, un cabaret pour nous signifier notre différence! Méfions nous de ces esprits de bonnes volontés, épris de colonialisme larvaire, au intentions salvatrices. Nous laissant comme seul issue, la résistance et la mobilisation ...Mr Cornut sachez sachez haut et fort et bien que le Négre vous emmerde vous ${ }^{24}$ et votre cabaret (Association kolors : 02/02/2017).

Le même positionnement de victime submergée par l'émotion se retrouve du côté de ceux qui dénoncent tous les dangers d'un monde en mouvement: «Ce Pauvre Monde est à l'agonie! Islamisme, Féminisme, Négritude, Multiculturalisme, Christamophobie, etc. Le réchauffement climatique leur monte à la tête!» (dufaitrez: 22/11/2017, https://ripostelaique.com/louis-georges-tin-veut-interdire-a-un-ecrivain-noir-deprendre-un-negre-blanc.html) L'hyperbole accompagne le rejet passionnel de ce que l'autre représente.

\section{Conclusion}

Qu'ils soient hostiles ou favorables à l'interdiction du mot " Nègre ", les protagonistes de ces conflits identitaires emploient des formes discursives assez proches. L'argumentation au sens technique joue un rôle minimal dans leur discours. L'essentiel repose tout d'abord sur une présentation de soi en victime, la posture de l'offensé valant droit à la parole; d'autre part, sur une présentation extrêmement négative des adversaires, les attaques ad hominem l'emportant sur les arguments ad rem. Aussi, les désignants de soi et des autres deviennent un enjeu considérable. Le mot qui vous nomme peut être infâmant et mériter le boycott (« Nègre »), ou il peut obliger la société à vous voir ("Noir ») et donner une visibilité à vos adversaires (« Blancs »).

Les militants du PIR, qui ont importé des problématiques universitaires américaines dans le débat européen, sont mobilisés en particulier contre le signifiant "Nègre ". L'Affaire du Bal Nègre/Bal Blomet a été un de ces épisodes. En s'alignant sur le combat américain contre Nigger, ils n'ont retenu que la valeur injurieuse du mot, négligeant son ambivalence historique, ses liens avec le créole et les connotations contextuelles positives qui l'associent aux mouvements artistiques du $20^{\mathrm{e}}$ siècle. Inversement les catégories de "Noirs", de "racisés», etc., transforment les "différences » ethnoraciales en étendard et permettent de demander des réparations au groupe dominant assigné à un statut de "Blancs » collectivement coupables devant l'histoire. Dans cette vision « racialiste », la mémoire de la domination et la couleur de peau priment sur les relations qui sont susceptibles de se nouer entre les habitants d'un territoire.

On retrouve sur les réseaux sociaux d'extrême droite la même division manichéenne de la réalité sociale en deux blocs identitaires. L'enjeu est analogue, pour paraphraser un titre de Luc Boltanski : « rendre la réalité inacceptable ». Cette fois, c'est le refus de voir la société se transformer en société multiculturelle qui conduit à fantasmer un passé homogène, une manière commune de vivre qui serait menacée par les nouveaux arrivants.

En s'opposant à la définition juridique du racisme, pour lui substituer une définition victimaire différencialiste, les militants de la cause "décoloniale", actifs sur les réseaux sociaux, se retrouvent au côté de ceux qui voient la société comme des blocs 
ethniques. Ils ajoutent des logiques d'exclusion et d'antagonisme communautaire à la lutte nécessaire contre les discriminations dans l'accès au logement et au travail.

\section{BIBLIOGRAPHIE}

Sources

Afrofem : https://afrofem.com/

Association Kolors. 2017. https://capoeirakolors.wordpress.com/2017/02/02/

nonaubaldescolons-pas-de-bal-negre-a-paris-en-2017/février 2017

Bal Blomet http://www.balblomet.fr/histoire-bal-blomet/

Césaire, Aimé. 2005. Nègre je suis, nègre je resterai, Aimé Césaire, entretien avec Françoise Vergès (Paris :

A. Michel)

Desnos, Robert. 1931. Comoedia https://gallica.bnf.fr/ark :/12148/cb32745939d/date1931.item

Eddo-Lodge, Reni. 2018 tr. Le Racisme est un problème de Blancs (Paris : Autrement)

Etats Généraux de l'Outre-Mer, 2009. http://archives.etatsgenerauxdeloutremer.fr/bannir-lemot-negre-du-vocabulaire.html

Hugo, Victor. 1826. Bug-Jargal (Paris : Canel)

Indigènes de la République http://indigenes-republique.fr/le-p-i-r/appel-des-indigenes-de-larepublique/

Léardée, Brigitte \& Jean-Pierre Meunier. 1989. La Biguine de l'Oncle Ben's : Ernest Léardée raconte (Paris : Editions caribéennes)

https://mwasicollectif.com/2017/02/06/manifestation-contre-le-bal-negre/

Riposte Laïque. 2017. https://ripostelaique.com/louis-georges-tin-veut-interdire-a-un-ecrivainnoir-de-prendre-un-negre-blanc.html

Sadaï, Célia. 2017. « Prochaine ouverture du Bal Nègre à Paris : quel usage du mot « nègre » en France? ", Africultures publié le 31 janvier 2017. http://africultures.com/prochaine-ouverturedu-bal-negre-a-paris-quel-usage-du-mot-negre-en-france-13955/

Références secondaires

2017. Argumentation et analyse du discours (AAD) 16, en ligne (https://journals.openedition.org/ $\mathrm{aad} / 2207)$

Boltanski, Luc. 2008. Rendre la réalité inacceptable. A propos de la production de l'idéologie dominante (Paris : Demopolis)

Bonnafous, Simone. 1991. L'immigration prise aux mots (Paris : Kimé)

Boutet, Josiane. 2010. Le Pouvoir des mots (Paris : La Dispute)

Butler, Judith. 2004. Le Pouvoir des mots. Politique du performatif, traduit de l'anglais (Excitable Speech, Routledge, 1997) par Charlotte Nordmann (Paris : Editions Amsterdam) 
Calabrese Laura \& Marie Veniard éds. 2018. Penser les mots. Dire la migration (Louvain-la-Neuve : Académia)

Charaudeau, Patrick. 2008. "L'argumentation dans une problématique de l'influence », Argumentation et Analyse du Discours1, L'analyse du discours au prisme de l'argumentation, en ligne (https://journals.openedition.org/aad/193)

Daget, Serge.1973. « Les mots esclave, nègre, Noir et les jugements de valeur sur la traite négrière dans la littérature abolitionniste française de 1770 à 1845 ", Revue française d'histoire d'Outre-Mer, XL, 221, 511-548. [https://www.persee.fr/doc/outre_0300-9513_1973_num_60_221_1711]

Debray, Régis. 1991. Cours de médiologie générale (Paris : Gallimard-Bibliothèque des Idées)

Delesalle, Simone \& Lucette Valensi. 1972. « Le mot 'nègre' dans les dictionnaires français d'Ancien régime ; histoire et lexicographie » Langue Française 15, 79-104. https://www.persee.fr/ doc/lfr_0023-8368_1972_num_15_1_5612

Ducrot, Oswald. 1984. Le Dire et le dit (Paris : Minuit)

Goffman, Erwin. 1975 tr. Stigmate : les usages sociaux des handicaps (Paris : Minuit)

Guillaumin, Colette. 1972. L'Idéologie raciste : genèse et langage actuel (La Haye : Mouton)

Guilhaumou, Jacques. 1991. L'Avènement des porte-paroles de la République (1789-1792). Essai de synthèse sur les langages de la Révolution (Arras : Septentrion)

Lecolle, Michéle, Marie Veniard \& Guérin Olivia éds. 2018. Langages 210, Vers une sémantique discursive : propositions théoriques et méthodologiques

Mbembé, Achille. 2000. De la postcolonie. Essai sur l'imagination politique dans l'Afrique contemporaine (Paris : Karthala)

Principato, Aurelio. 2016. «La réversibilité identitaire », Salerni, Paola \& Jörg Senf, Textes et contextes de l'immigration (Paris : Hermann) 164-176

Saïd, Edward. 1978 tr. L'orientalisme. L'orient créé par l'occident (Paris : Seuil)

Searle, John R. 1998 tr. [1995]. La Construction sociale de la réalité (Paris : Gallimard. Essais)

Sue, D.W. et al. 2007. "Racial microaggressions in everyday life : Implications for clinical practice" , American Psychologist 62(4), 271-286

Tarde, Gabriel. 1979 [1895]. Les lois de l'imitation (Paris-Genève : Slatkine)

\section{NOTES}

1. Quand le compte a été fermé, il avait recueilli 7541 signatures.

2. En $\mathrm{AD}$, de nombreux chercheurs ont travaillé à partir d'entrées lexicales autour de différentes minorités. Voici quelques références : Bonnafous (1991) sur les désignants des migrants dans un corpus de presse, ou le numéro 116 de Mots (2018). Plus largement, sur la violence symbolique exercée par les mots, on pourra lire Butler (2004) et de nombreux exemples dans Boutet (2010). Pour une perspective qui insiste sur les aspects dynamiques du lexique,voir le $\mathrm{n}^{\circ} 210$ de Langages, coordonné par M. Lecolle et al. (2017), ainsi qu'à l'ouvrage coordonné par Calabrese et Veniard (2018). Sur le lien entre nomination et analyse argumentative, voir le $\mathrm{n}^{\circ} 17$ de la revue Argumentation et Analyse du Discours en particulier l'introduction de R. Koren. 
3. Indigènes est la reprise ironique d'un terme qui renvoyait à l'inégalité juridique des populations natives des colonies, constituées de « sujets » français et pourtant exclues de la citoyenneté. Avec ce vocabulaire, les fondateurs de ce mouvement affirment le continuum du fait colonial et de la vie inégalitaire dans la France contemporaine. Voir http://indigenes-republique.fr/le-p-i-r/appel-des-indigenes-de-la-republique/

4. Le courant postcolonial bien développé dans le monde universitaire anglo-saxon critique les prétentions universalistes de l'Occident à partir du constat de la violence qui s'est exercée contre les colonisés et des traces de domination qui perdurent dans la société. Voir le livre fondateur de Saïd (1978) et Mbembé (2000).

5. Le CRAN, Conseil Représentatif des Associations Noires de France, a été créé le 26 novembre 2005, à l'Assemblée nationale.

6. L'ouvrage a été rapidement couronné par des prix en Grande-Bretagne (notamment en 2018 le British Book Award for a Work of Narrative Non-Fiction).

7. Voir, par exemple, l'Essai sur les Connaissances Humaines de Condillac (1746) qui explique le développement des facultés humaines et notamment du langage par le fait que les individus ne sont pas enfermés en eux-mêmes, mais identifient les émotions de l'Autre comme les leurs propres.

8. Ce que les universitaires américains ont pris l'habitude de désigner comme des micro-agressions. Sue (2007).

9. Pour reprendre l'expression de Boltanski (1990 : 40-41).

10. Voir l'introduction de Guilhaumou à son livre de 1991, dont la thématique n'est pas sans rappeler les phénomènes que j'évoque ici.

11. Guilhaumin (1972) introduit cette perspective américaine en France en réservant le terme à la construction sociohistorique de la " race ", par opposition aux conceptions racistes qui inscrivent l'identité des individus dans un règne naturel.

12. Pour Charaudeau (2008) l'analyse du discours n'a pas à se donner pour objet la découverte de la Vérité, mais la découverte des jeux de mise en scène de la vérité comme « croire » et « faire croire ». C'est ce que j'appelle une problématique de l'influence.

13. Comoedia est un journal, aujourd'hui disparu, qui a paru avec une périodicité variable entre 1907 et 1944. Les numéros sont consultables sur Gallica, les archives numériques de la BNF. Je cite Desnos en me fiant aux renseignements fournis par le Wikipedia à l'article Bal Nègre (https://fr.wikipedia.org/wiki/Bal_N\%C3\%A8gre=) Je n'ai pas réussi à retrouver la citation de Desnos dans les exemplaires de Comoedia que j'ai consultés.

14. Pétition adressée à Guillaume Cornut, Anne Hidalgo Maire de Paris, Philippe Goujon Maire de Paris 15e et Audrey Azoulay Ministre de la Culture.) https://www.change.org/ p/nonaubaldescolons-pas-de-bal-n\%C3\%A8gre-\%C3\%A0-paris-en-2017 (suit la liste des premiers signataires)

15. Il s'est apparemment contenté de quelques échanges, notamment avec l'écrivain Claude Ribbe qui en dément le contenu.

16. Qui ont eux-mêmes tirés un substantif de la racine adjectivale latine niger (noir, sombre).

17. Sur cette inversion des responsabilités, voir Delesalle et Valensi (1972).

18. Daget (1973) montre qu'en 1845 encore, les abolitionnistes sous-emploient « traite des Noirs » et emploient normalement « traite des nègres ».

19. Le renversement des valeurs a ses limites et les frères esclaves de Bug-Jargal n'ont pas ses qualités chevaleresques (81-97). 
20. Son inspiration, puisée dans ses expériences de marin, est plutôt précolombienne. En 1891, il s'installe à Tahiti.

21. Africultures est une revue fondée en 1997, consacrée aux arts et à la culture en provenance d'Afrique et de ses diasporas.

22. Depuis les travaux de Ducrot (1984), on est sensible à l'interprétation polémique des énoncés négatifs. Si l'on considère en contexte certains énoncés assertifs, on s'aperçoit que, disant, ils s'opposent à tout le paradigme des énoncés qui auraient pu apparaître à leur place, produisant le même type d'effet polémique.

23. De fait, les Français semblent importer les batailles qui ont eu lieu aux Etats-Unis autour de Malcolm X et qui ont entraîné le rejet de Nigger au profit de Black ou d'AfroAmerican.

24. Allusion à la phrase célèbre d'Aimé Césaire (2005) : « Le nègre vous emmerde ». « Nègre je suis, nègre je resterai ».

\section{RÉSUMÉS}

Cet article examine un bref moment discursif où, en 2017, des militants antiracistes «différentialistes » se sont battus pour obtenir le changement de nom d'un théâtre, «Le Bal Nègre » (en mémoire du nom qui l'a rendu célèbre dans les années 30), affirmant que, quel que soit le contexte linguistique, « Nègre » est une insulte. J'analyserai certains aspects du discours de ceux qui se considèrent comme victimes du racisme néocolonial et du discours des groupes qui se sentent injustement accusés et qui se décrivent comme les victimes d'un nouveau racisme inversé. Adoptant, un point de vue discursif qui part du fait que le langage est utilisé pour construire des images sociales de soi et de l'autre, ce travail cherche à dégager des caractéristiques représentatives du discours antiraciste actuel. Il explore la façon dont l'énonciation des victimes articule expérience intime et généralisation, émotion et argumentation; le rôle du scandale: l'événement, même minime, renforce le sentiment d'appartenance à une force collective; les batailles autour des dénominations sociales; la construction d'une démarcation binaire entre des catégories qui efface toute hétérogénéité interne; le rôle des émotions partagées sur les réseaux sociaux qui consolident le sentiment d'appartenance à un groupe de victimes.

This article examines a brief discursive moment when, in 2017, "differentialist" anti-racist activists fought to change the name of a theater, "The Bal Nègre" (in memory of the name that made it famous in the 1930s), arguing that, regardless of the linguistic context, "Nègre" (Negro) is an insult. I will analyze some aspects of the discourse of those who see themselves as victims of neocolonial racism, as well as of those who feel unjustly accused and who describe themselves as victims of a new inverted racism. Adopting a discursive point of view emphasizing that language is used to construct social images of the self and the other, I seek to identify some characteristics of the current anti-racist discourse: the enunciation of the victims articulates intimate experience and generalization, emotion and argumentation; the role of scandal: the event, however small, reinforces the feeling of belonging to a collective force; battles around corporate names; the construction of a binary demarcation between black and white( highlighting 
similarities and eliminating differences); the role of emotions shared on social networks that consolidate the feeling of belonging to a group of victims.

\section{INDEX}

Mots-clés : analyse du discours, Bal Nègre, Nègre/Noir, nomination conflictuelle, victime Keywords : Bal Nègre, conflictual nomination, discourse analysis, Nègre/Black, victim

\section{AUTEUR}

\section{SONIA BRANCA-ROSOFF}

Université Sorbonne Nouvelle, Paris 3 